\title{
Contribution of the Dorsal Nucleus of the Lateral Lemniscus to Binaural Responses in the Inferior Colliculus of the Rat: Interaural Time Delays
}

\author{
Sean A. Kidd and Jack B. Kelly \\ Laboratory of Sensory Neuroscience, Institute of Neuroscience and Department of Psychology, Carleton University, \\ Ottawa, Ontario, Canada K1S 5B6
}

The contribution of the dorsal nucleus of the lateral lemniscus (DNLL) to binaural responses in the inferior colliculus of the rat was determined for a wide range of interaural time differences (ITDs). Single-unit action potentials were recorded from the inferior colliculus before and after local injection of the excitatory amino acid antagonist kynurenic acid into the DNLL. Binaural properties were determined by manipulating the time difference between paired clicks delivered to the ears ipsilateral and contralateral to the recording site. The probability of an action potential decreased as contralateral stimulation was delayed, relative to ipsilateral stimulation. These data generated a sigmoidal ITD curve for delays between -1.0 and +1.0 msec. By extending the time intervals beyond $1 \mathrm{msec}$, it was possible to determine the trailing edge of the inhibition pro- duced by ipsilateral stimulation. The duration of the inhibitory effect varied from cell to cell but lasted as long as $20 \mathrm{msec}$ in some cases. Injection of kynurenic acid into the DNLL contralateral to the recording site reduced the extent of both short (0-1 msec) and long-lasting (1-20 msec) inhibition in the inferior colliculus. No effect was seen after injections ipsilateral to the recording site. The data demonstrate that the DNLL plays an important role in shaping ITD responses in the inferior colliculus and contributes to both the short and long-lasting inhibition produced by stimulation of the ipsilateral ear.

Key words: binaural processing; sound localization; precedence effect; auditory spatial perception; interaural time difference
The dorsal nucleus of the lateral lemniscus (DNLL) is a binaurally responsive GABAergic nucleus of the auditory midbrain with prominent projections to both the ipsilateral and contralateral central nucleus of the inferior colliculus and the contralateral DNLL (Brugge et al., 1970; Beyerl, 1978; Adams, 1979; BrunsoBechtold et al., 1981; Kudo, 1981; Zook and Casseday, 1982; Adams and Mugnaini, 1984; Tanaka et al., 1985; Thompson et al., 1985; Coleman and Clerici, 1987; Moore and Moore, 1987; Roberts and Ribak, 1987; Ross et al., 1988; Shneiderman et al., 1988, 1993; Oliver and Shneiderman, 1989; Shneiderman and Oliver, 1989; Hutson et al., 1991; Vater et al., 1992; Bajo et al., 1993; Buckthought, 1993; Covey, 1993; Markovitz and Pollak, 1994; Merchán et al., 1994; Winer et al., 1995). Electrophysiological studies have shown that the DNLL is a source of inhibition affecting binaural responses in the inferior colliculus of the rat. Pharmacological blockade of the DNLL by injection of the glutamate antagonist kynurenic acid (Li and Kelly, 1992b) or other substances (Faingold et al., 1993) alters the binaural characteristics of neurons in the contralateral inferior colliculus. Specifically, the binaural inhibition produced by varying the interaural intensity difference (IID) of tones delivered simultaneously to the two ears is reduced by injection of antagonists into the DNLL contralateral to the recording site.

\footnotetext{
Received July 3, 1996; revised Aug. 27, 1996; accepted Aug. 30, 1996.

This research was supported by the Natural Sciences and Engineering Research Council of Canada. We thank Rosalie Labelle and Brian van Adel for their generous contribution of time and experience to this study.

Correspondence should be addressed to Dr. Jack B. Kelly, 329 Life Science Building, Laboratory of Sensory Neuroscience, Carleton University, 1125 Colonel By Drive, Ottawa, Ontario, Canada K1S 5B6.

Copyright (C) 1996 Society for Neuroscience $0270-6474 / 96 / 167390-08 \$ 05.00 / 0$
}

The binaural neurons in the inferior colliculus of the rat are also sensitive to interaural time differences (ITDs). Although most neurons in the inferior colliculus of the rat are insensitive to ongoing binaural phase differences (Kelly et al., 1991), they are nevertheless very sensitive to the time differences between transients delivered to the two ears. Binaural time sensitivity has been demonstrated previously for neurons in the auditory cortex of the rat by using clicks rather than tone bursts to elicit single-unit responses (Kelly and Phillips, 1991), and Glenn and Kelly (1992) have shown that unilateral kainic acid lesions of DNLL alter ITD curves derived from surface-evoked potentials in the contralateral auditory cortex. However, so far there have been no systematic studies of the influence of DNLL on ITD sensitivity of neurons in the inferior colliculus. The main purpose of the present study was to determine the contribution of DNLL to the ITD sensitivity of single neurons in the inferior colliculus of the rat.

Of particular interest for the study of ITD sensitivity is the presence of inhibition in the inferior colliculus that persists for many milliseconds after the initiating acoustic stimulus. It has been suggested that this inhibition originates in the DNLL and might be related to the perceptual suppression of echo (Carney and Yin, 1989; Yang and Pollak, 1994 a,b; Yin, 1994; Fitzpatrick et al., 1995). One objective of the present study was to examine the possible contribution of the DNLL to long-lasting inhibition in the inferior colliculus of the rat.

We have determined the effects of pharmacological blockade of DNLL on responses in inferior colliculus to both short and long ITD intervals. Data were obtained before and after kynurenic acid injections into the ipsilateral or contralateral DNLL. 


\section{MATERIALS AND METHODS}

Physiological procedures. Electrophysiological experiments were conducted on 24 male Wistar albino rats $(250-500 \mathrm{gm})$ purchased from Charles River (St. Constant, Quebec). The animals initially were anesthetized with sodium pentobarbital $(60 \mathrm{mg} / \mathrm{kg}$, i.p.) to induce surgical anesthesia. Then they were maintained in an areflexive state during the recording sessions by supplemental injections of Equithesin $[0.5 \mathrm{ml} / \mathrm{kg}$; see Li and Kelly (1992a) or Sally and Kelly (1992) for preparation]. Before surgery, the auditory canal was inspected with an otoscope and found to be free of obstruction. The animals were placed in a head holder that left the external meatus open for subsequent insertion of earphone drivers. A midline incision was made in the scalp, and the tissue was retracted laterally to expose the skull. Small craniotomies were made to allow insertion of recording and injection pipettes into the inferior colliculus and the DNLL, respectively.

Coordinates for positioning the recording and injection pipettes were referenced from lambda with the skull flat (Paxinos and Watson, 1986). For placements in DNLL, the pipette was tilted $30^{\circ}$ against the sagittal plane and lowered into the brain from a point $6.7 \mathrm{~mm}$ lateral and $0.4-0.5$ $\mathrm{mm}$ rostral to lambda. The pipette was lowered to a depth of $7.8 \mathrm{~mm}$, and its position was adjusted to obtain physiological responses from DNLL. The injection pipette itself served as a recording electrode to monitor the neural activity in DNLL, as described previously by Li and Kelly (1992b), and the position of the pipette could be fine-tuned to give the best acoustically driven response. Also, the blocking effect of the kynurenic acid on neural responses in DNLL could be monitored directly during and after the injection.

The injection pipettes were pulled from single-barrel glass tubing [1.0 $\mathrm{mm}$ outer diameter (o.d.), $0.5 \mathrm{~mm}$ inner diameter (i.d.); Sutter Instruments, Novato, CA] to a tip diameter of $20-40 \mu \mathrm{m}$. The pipettes were backfilled with $2 \mathrm{~mm}$ kynurenic acid in Locke's solution, and a tungsten wire was inserted into the solution to provide contact for electrical recordings. Pipettes were connected by a length of flexible tubing to a 5 $\mathrm{ml}$ syringe for pressure injections. The volume of the injection $(2.0-2.5$ $\mu \mathrm{l})$ was controlled by monitoring the progression of the solution along the length of the injection pipette and stopping the flow by releasing the pressure through a three-way stopcock.

Recording pipettes were inserted into the inferior colliculus either obliquely $\left(30^{\circ}\right.$ against the sagittal plane) or vertically, following stereotaxic coordinates. For oblique penetrations, the pipette was positioned $0.4 \mathrm{~mm}$ rostral and $4 \mathrm{~mm}$ lateral to lambda and lowered to a depth of at least $5 \mathrm{~mm}$. For vertical penetrations the pipette was positioned $0.4 \mathrm{~mm}$ rostral and $2.0 \mathrm{~mm}$ lateral to lambda and was lowered to a depth of $3.0-5.0 \mathrm{~mm}$. In either case the pipette position was adjusted to obtain short latency accoustically driven responses that exhibited narrow band frequency tuning and a clearly defined characteristic frequency $(\mathrm{CF})$. The $\mathrm{CF}$ of neurons in the inferior colliculus showed a progressive low-to-high frequency gradient as the recording pipette was lowered dorsoventrally through the central nucleus. Histological reconstructions confirmed the location of electrode placements in the central nucleus of the inferior colliculus.

Recording pipettes were pulled from single-barrel glass tubing $(1.0 \mathrm{~mm}$ o.d., $0.5 \mathrm{~mm}$ i.d.; Sutter) to a tip diameter of $\sim 2 \mu \mathrm{m}$. They were backfilled with Locke's solution (1.5-2.5 M $\Omega$ ) and connected to recording equipment via an insulated tungsten electrode. Physiological potentials were amplified by Dagan EX4-400 amplifiers, displayed on oscilloscopes, and monitored acoustically by a loudspeaker. Neural responses were digitized and processed by MALab 881, a data acquisition system designed and produced by Steve Kaiser, Department of Neurobiology, University of California, Irvine (Kaiser Instruments) for use with Macintosh computers (in our case, a Quadra 700). The program provided a digital window discriminator for selection of action potentials and displayed poststimulus time histograms and other data on-line. Physiological responses were stored on optical disk and processed later with standard database and graphics software.

Stimulus parameters. Sounds were presented separately to the two ears through headphone drivers (Pioneer SE-50D) mounted in sealed housings that were fitted to hollow couplers inserted into the external meatus of the rat. Sounds were generated digitally by a Kaiser Instruments DA interface controlled by MALab 881 to produce either tone pulses (100 msec with $10 \mathrm{msec}$ rise and fall times) or clicks (50 $\mu \mathrm{sec}$ square waves). The clicks had a broad spectrum from 0.1 to $25 \mathrm{kHz}$, essentially flat up to $4.0 \mathrm{kHz}$ and rolling off at higher frequencies. The sound pressure of tone pulses was referenced to a threshold of a cell at $\mathrm{CF}$, and the sound pressure of clicks was calibrated in decibels of sound pressure level (SPL; re 0.0002 dynes $/ \mathrm{cm}^{2}$ ) by using a $1 / 2$ inch $B \& K$ microphone with the headphone speculum inserted into an enclosed cavity. For most experiments the SPL of clicks delivered to either ear was set at $20 \mathrm{~dB}$ above the threshold for eliciting a response by monaural stimulation of the contralateral ear.

All recordings were obtained from well isolated single units defined by the presence of action potentials of constant amplitude and waveform. Before investigating responses to binaural time differences, we examined the neural response to tone pulses. First, the characteristic frequency (CF, the frequency to which a neuron responded at the lowest SPL) was determined with monaural stimulation of the contralateral ear. Then the binaural response pattern to tonal stimulation was determined by setting the contralateral stimulus level at $20 \mathrm{~dB}$ above threshold and presenting ipsilateral tone pulses simultaneously in steps of increasing intensity. In some cases, both ipsilateral and contralateral stimulation produced excitation, and combined stimulation resulted in facilitation. The vast majority of cells, however, showed binaural suppression, i.e., the contralateral response was strongly inhibited by simultaneous ipsilateral stimulation. In some cases a slight facilitation occurred at low levels of ipsilateral stimulation, but strong response suppression was seen as the ipsilateral level was increased. Both types of binaural suppression are referred to here as EI (excitatory-inhibitory) responses (Kelly et al., 1991; Li and Kelly, 1992b). The present report is based exclusively on neurons with EI binaural response patterns.

After responses to tone bursts had been recorded, the neurons were tested with clicks to determine their response to binaural time differences. The clicks were delivered to the ears at various interaural time intervals, with each interval repeated 30 times at a rate of $1 / \mathrm{sec}$. Initially we focused our attention on intervals between -1.0 and $+1.0 \mathrm{msec}$, a range that spans the interaural differences produced by a single sound source located at various free-field positions in the azimuthal plane. We then investigated much larger time intervals from -1.0 to $+30.0 \mathrm{msec}$ to determine the persistence of inhibition produced by stimulation of the ipsilateral ear. For each neuron the same range of binaural time intervals was explored before and after injection of kynurenic acid into the DNLL.

Four animals (6 neurons) were used to provide normative data on the effects of varying the intensity as well as the timing of ipsilateral stimulation. In these cases, both short and long ITD intervals were presented with the ipsilateral sound pressure set at different levels. The purpose of these experiments was to determine the extent to which ITD sensitivity was affected by ipsilateral level. All experiments with kynurenic acid injections into DNLL, however, were done with the ipsilateral and contralateral levels equal and invariant.

Histology. After completion of a recording session, a solution of brilliant blue $\mathrm{G}(0.25 \%$ in distilled water) was added to both the recording and the injection pipettes while they were still in position in the brain. Then the dye was ejected from the pipette tip iontophoretically by applying negative current $(8-10 \mu \mathrm{A}, 20-30 \mathrm{~min})$ to provide a visible marker for histological verification of recording and injection sites. In preparation for histology, the animals were given a large injection of sodium pentobarbital $(120 \mathrm{mg} / \mathrm{kg}$, i.p.) and perfused transcardially with $0.1 \mathrm{~m}$ PBS, followed by $10 \%$ formalin. The brains were removed, stored in a $20 \%$ sucrose $/ 10 \%$ formalin solution, and cut serially at $40 \mu \mathrm{m}$ in the frontal plane on a freezing microtome. The location of pipette tracks and dye deposition at the pipette tips was determined microscopically from unstained sections.

All data presented here were obtained from cases in which the injection pipette was directly in DNLL and the recording pipette was in the central nucleus of the inferior colliculus.

\section{RESULTS}

\section{Normal response}

The effects of ITD were determined for 27 single neurons in the inferior colliculus of the rat. Data for three typical neurons are presented in Figure 1 to illustrate the effects of ipsilateral stimulation on short and long ITDs. Several general points emerge from these examples. First, virtually every EI neuron tested was sensitive to binaural time differences, regardless of its $\mathrm{CF}$. Although most neurons in the inferior colliculus of the rat have CFs above $1.0 \mathrm{kHz}$ and are completely insensitive to ongoing phase differences, they are nevertheless highly sensitive to the time differences between transients such as clicks. For each of the 

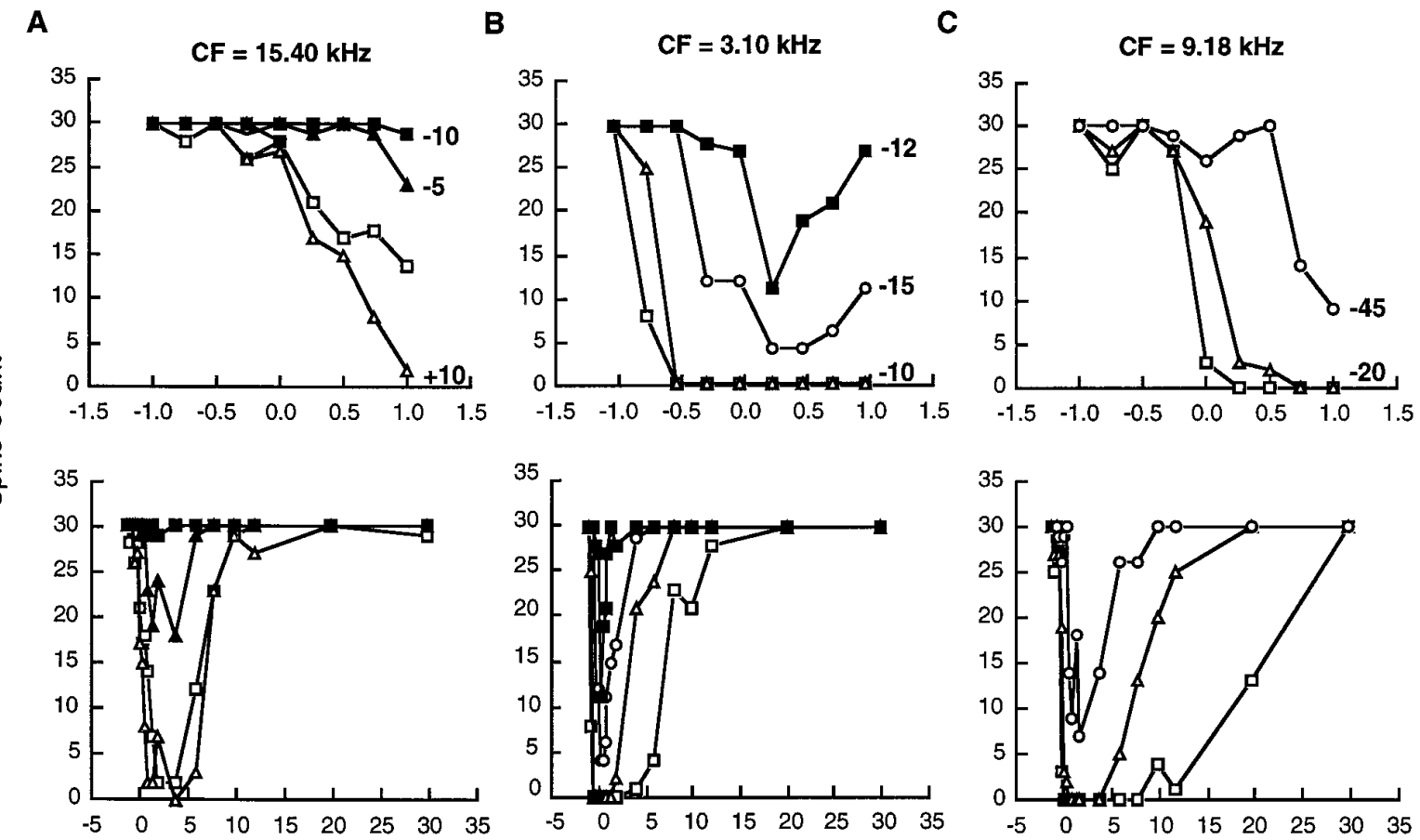

ITD (ipsi re contra, ms.)

Figure 1. ITD curves for three neurons $(A-C)$ in the inferior colliculus with contralaterally determined CFs of $15.40,3.10$, and $9.18 \mathrm{kHz}$. Separate ITD curves were recorded at different ipsilateral sound pressure levels (SPL) with contralateral sound pressure held constant at $20 \mathrm{~dB}$ above threshold (absolute values were 81, 87, and $67 \mathrm{~dB}$ SPL for neurons $A-C$, respectively). The ITD curve obtained with the ipsilateral and contralateral intensity equal is represented by open squares (IID $=0 \mathrm{~dB}$ ). Other curves are plotted for various ipsilateral sound pressure levels above and below $0 \mathrm{~dB}$, as indicated in the graph. The top three panels present data for ITD intervals between -1.0 and $+1.0 \mathrm{msec}$. The bottom three panels present data from the same cells for larger ITD with ipsilateral lead times up to $30 \mathrm{msec}$. The number of spikes in this and all other figures is based on 30 stimulus presentations.

neurons shown here with CFs of $3.10,9.18$, and $15.40 \mathrm{kHz}$, the number of action potentials over 30 trials dropped systematically as the contralateral stimulus was delayed, relative to the ipsilateral stimulus. The strength of ipsilateral inhibition increased monotonically over the range of ITDs from -1.0 to $+1.0 \mathrm{msec}$. The strength and time course of ipsilateral inhibition were also affected by the intensity of ipsilateral stimulation. Lowering ipsilateral sound pressure invariably reduced the inhibitory effect. For some neurons, ipsilateral inhibition was near saturation at equal sound pressure of the clicks presented to the two ears. For others, the strength of inhibition increased further with an additional increase in the level of ipsilateral stimulation. The time interval of maximum sensitivity as defined by the slope of the ITD curve varied somewhat from cell to cell. For example, the cells illustrated in Figure $1 A-C$ were most sensitive at approximately +0.5 , -0.5 , and $0.0 \mathrm{msec}$, respectively.

The long-lasting inhibition produced by ipsilateral stimulation is shown for the same three cells in the bottom panels of Figure $1 A-C$. Data are plotted over an ITD range from -1.0 to $30 \mathrm{msec}$, with positive values referring to an ipsilateral-leadingcontralateral click order. Each of these neurons showed evidence of long-lasting inhibition, regardless of CF. However, the time course of the inhibition was different from cell to cell. The inhibitory effect lasted 10,15 , and up to $20 \mathrm{msec}$ for the cells illustrated in Figure $1 A-C$, respectively. The extent of inhibition and the length of the inhibitory period were greatly affected by the intensity of the ipsilateral stimulus. The extent of inhibition was reduced as the ipsilateral intensity was lowered. It should be pointed out that we found no systematic relation between the length of the inhibitory period and the CF of the neuron. Inhibitory periods as long as $20 \mathrm{msec}$ or as short as 5 or $10 \mathrm{msec}$ were found routinely in neurons with either high or low CFs.

\section{Kynurenic acid injections}

The effect of injecting kynurenic acid into the contralateral DNLL was determined for 17 inferior colliculus neurons. Results are illustrated in Figure 2 for the response of four neurons to short ITDs. In each of the four cases, kynurenic acid injection resulted in a release from the inhibition produced by stimulation of the ipsilateral ear, and ITD sensitivity curves were affected over the range of +1.0 to $-1.0 \mathrm{msec}$. In two cases (cells $B$ and $D$ ), ITD curves were reexamined $1 \mathrm{hr}$ after injection of kynurenic acid. The strength of inhibition and the shape of the ITD curve returned to normal after this recovery period. Thus, the pharmacological blockade of neural activity in DNLL produced a reversible reduction in the inhibition associated with ipsilateral stimulation and altered the ITD sensitivity of neurons in the contralateral inferior colliculus.

Data were obtained from eight neurons for both short and long ITD intervals. The results of these experiments are summarized in Figure 3 for six representative cells. The data for short ITD intervals between -1.0 and $+1.0 \mathrm{msec}$ (except in $D$ ) are plotted in the top panels, and those for longer ITD intervals between -1.0 and 20-30 msec are plotted in the bottom panels for each of these cells. With short ITDs, injection of kynurenic acid into the DNLL contralateral to the recording site had a pronounced effect on the 

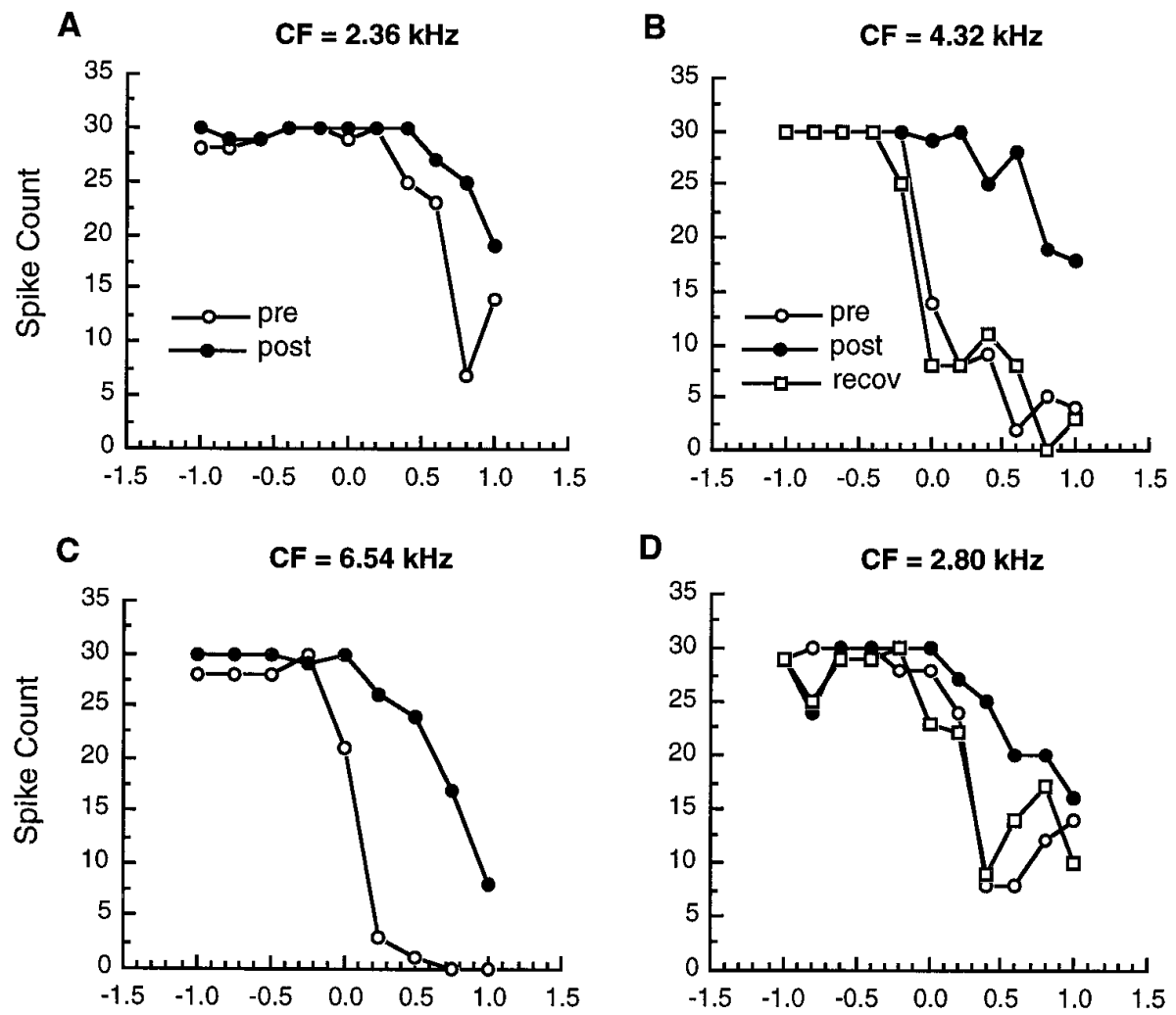

\begin{abstract}
Figure 2. The effect of injecting kynurenic acid into the DNLL on responses in inferior colliculus for ITDs between -1.0 and $+1.0 \mathrm{msec}$. All injections were made into the DNLL contralateral to the recording site located in the central nucleus of the inferior colliculus. Open circles represent responses before injection, and filled circles show responses after the injection. Open squares shown in $B$ and $D$ represent responses obtained after a recovery period of at least $1 \mathrm{hr}$.
\end{abstract} ITD (ipsi re contra, ms.)

response of the cell. For each neuron there was a release from the inhibition produced by stimulation of the ipsilateral ear. For one case (illustrated in $C$ ), complete recovery of the ITD curve was demonstrated after a postinjection period of $\sim 1 \mathrm{hr}$.

For each of these neurons, the injection of kynurenic acid into DNLL also affected responses to longer ITD intervals. Before injection the neurons exhibited ipsilaterally induced inhibitory periods that lasted 5-10 msec in the case of cells $A$ and $B, 15-20 \mathrm{msec}$ in cell $C$, and $>20 \mathrm{msec}$ in the case of cells $D-F$. After the DNLL injection, the strength of this long-lasting inhibition was reduced throughout the entire ITD period. These data show that neural activity in the contralateral DNLL is important for the expression in inferior colliculus of longlasting inhibition associated with stimulation of the ipsilateral ear.

Injections ipsilateral to the recording site had no effect on binaural responses in the inferior colliculus. Two examples are shown in Figure 4 for neurons tested with equal sound pressure in the two ears (IID $=0 \mathrm{~dB}$ ). One cell, with a $\mathrm{CF}$ of $5.50 \mathrm{kHz}$, was sensitive to small ITDs between 0.0 and $1.0 \mathrm{msec}$ and exhibited a long-lasting inhibition with ipsilateral lead times up to $\sim 8 \mathrm{msec}$. Kynurenic acid injection into the ipsilateral DNLL had no effect on the response of the cell to either short or long ITD intervals. The other cell, with a CF of $12.2 \mathrm{kHz}$, was sensitive to ITDs between -0.1 and 0.4 and exhibited a prolonged inhibition with ipsilateral lead times up to $30 \mathrm{msec}$. In this cell, also, there was no obvious effect of kynurenic acid injection on either short- or long-duration inhibition.

The effects of both ipsilateral and contralateral DNLL injections on long-lasting inhibition were examined in a single animal by recording simultaneously from neurons in the left and right inferior colliculus ( $\mathrm{CF}=12.58$ and $5.80 \mathrm{kHz}$, respectively). The data are shown in Figure 5 for ITDs between -1.0 and $20 \mathrm{msec}$. Before DNLL injections, both neurons exhibited a pronounced inhibition at ipsilateral lead times up to $20 \mathrm{msec}$. After injection of kynurenic acid into the left DNLL, the long-lasting inhibition in the right (contralateral) inferior colliculus was greatly reduced but inhibition over the corresponding ITD period in the left inferior colliculus was unaffected. After these data had been obtained, a second injection of kynurenic acid was made into the right DNLL. The interval between the first and second injection was $\sim 10 \mathrm{~min}$, insufficient for recovery to take place. Thus, the effect of the second injection was cumulative with the first. After the second injection there was a release from long-lasting inhibition of the neuron in the left (contralateral) inferior colliculus, but no additional effect was seen on the response of the neuron in the right inferior colliculus.

\section{DISCUSSION}

The results of this study show that binaural neurons in the inferior colliculus of the rat are sensitive to ITDs over the range of -1.0 to $+1.0 \mathrm{msec}$. For EI neurons, which constitute by far the largest population of acoustically responsive cells in the central nucleus of the inferior colliculus of the rat (Flammino and Clopton, 1975; Silverman and Clopton, 1977; Kelly et al., 1991), monaural stimulation of the contralateral ear excites and simultaneous stimulation of the ipsilateral ear strongly inhibits the production of action potentials. With transient stimuli (clicks), binaural time differences that favor the contralateral ear (contralateral-leadingipsilateral stimulation) result in a high probability of responding, a single action potential being evoked for every stimulus presented under the conditions of the present study. As the binaural 

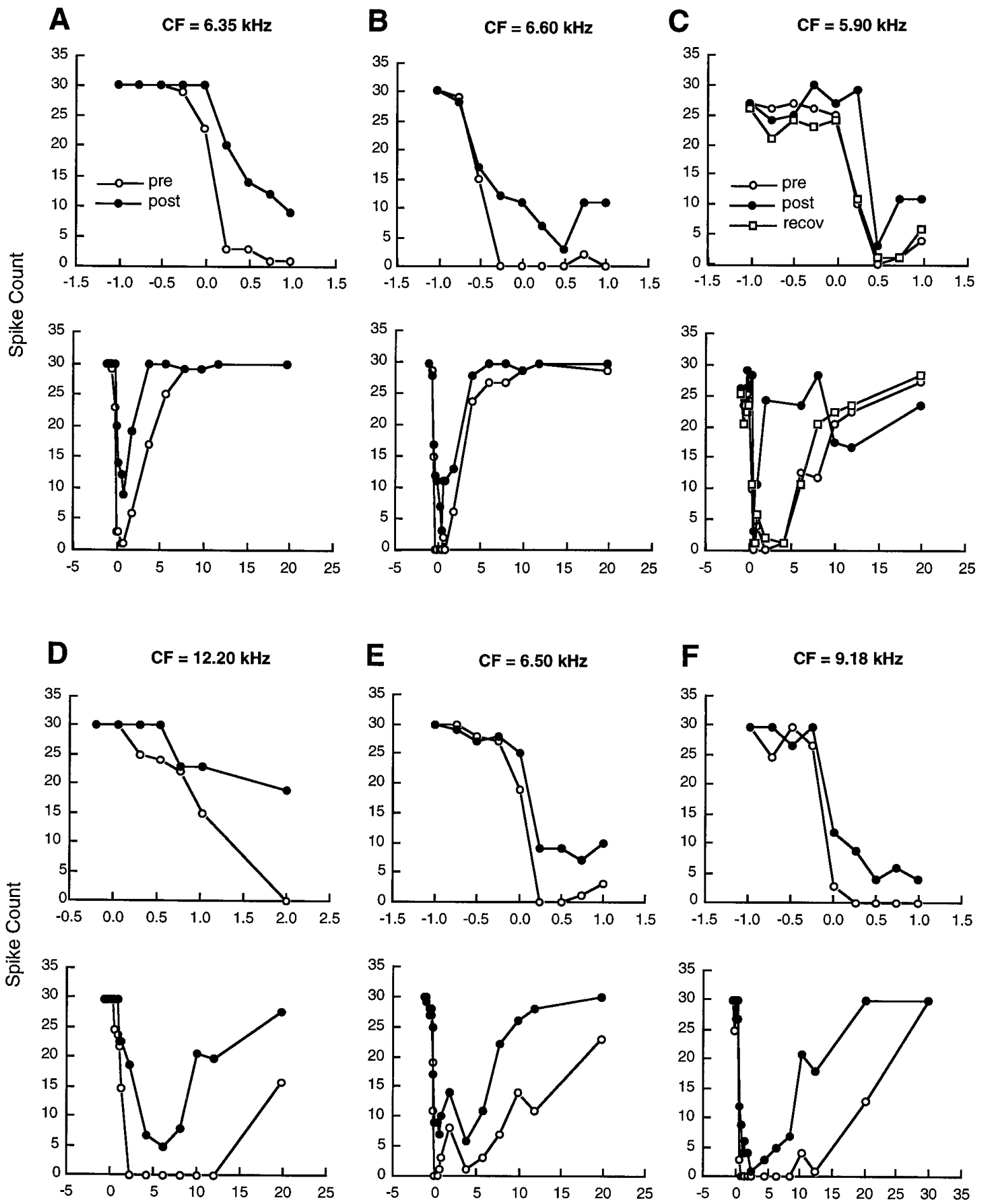

ITD (ipsi re contra, ms.)

Figure 3. The effect of kynurenic acid injection on responses to both short and long ITD intervals. Injections were made into the DNLL contralateral to the recording site in inferior colliculus. The top panels for neurons $A-F$ show responses for ITDs between -1.0 and +1.0 msec, with the exception of neuron $D$ for which the axis has been shifted by $1.0 \mathrm{msec}$. The bottom panels show ITDs between -1.0 and +20 msec, with the exception of cell $F$ for which the axis has been extended to $30 \mathrm{msec}$. Symbols are the same as in Figure 2.

time difference is shifted in favor of the ipsilateral ear, however, the response probability progressively drops to values near zero. The dynamic portion of the ITD curve is usually centered $\sim 0$ $\mu \mathrm{sec}$, although in some cases it is shifted to values above or below zero. The mean dynamic range of inferior colliculus neurons, as estimated from the 90 and $10 \%$ points on the ITD curve, with equal levels of stimulation in the two ears was $0.57 \mathrm{msec}$, which compares favorably with $0.59 \mathrm{msec}$ for primary auditory cortical 

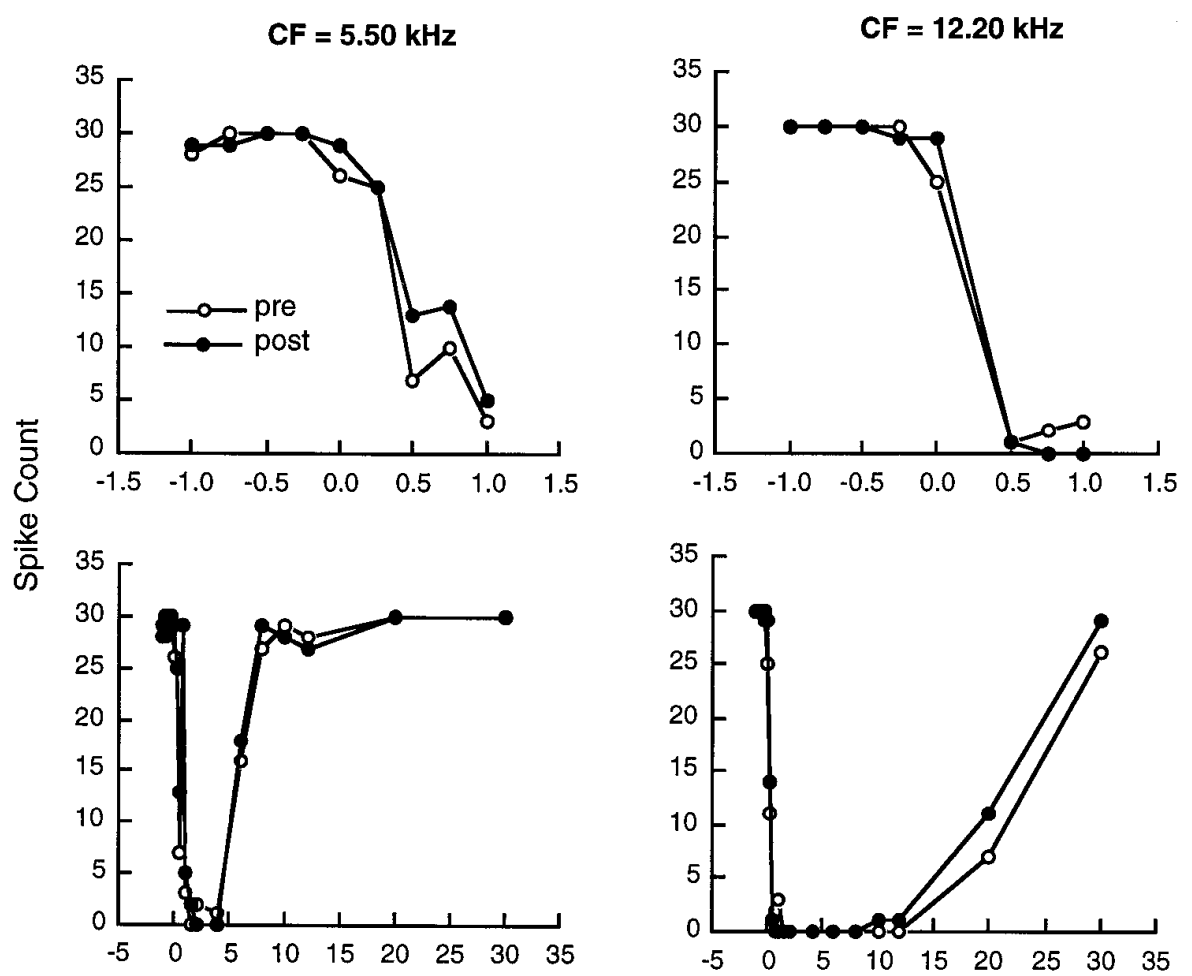

Figure 4. The apparent lack of effect of kynurenic acid injection into the DNLL ipsilateral to the recording site in inferior colliculus. Top panels show responses to ITDs between -1.0 and $+1.0 \mathrm{msec}$, and bottom panels show responses to ITDs between -1.0 and $+30 \mathrm{msec}$. Open circles represent responses before the injection, and filled circles represent responses af-

ITD (ipsi re contra, ms.) ter the injection.

neurons recorded previously in rats under similar experimental conditions (Kelly and Phillips, 1991).

The dynamic range of ITD curves for most neurons in the inferior colliculus of the rat is considerably larger than the binaural time differences associated with single sounds presented in the free field. The adult rat's head has an interaural distance of $\sim 3.0$ $\mathrm{cm}$. Based on a spherical model for calculating ITD, the maximum time difference generated by a sound located on the left or right would be $\pm 130 \mu \mathrm{sec}$, although predictions based on the shortest path across the rat's head yield a somewhat smaller value (116 $\mu \mathrm{sec})$. Therefore, only a relatively small portion of the dynamic range could possibly be used for encoding sound source position (see Kelly and Phillips, 1991). Nevertheless, given that ITDsensitive neurons respond differently to sounds located on the left or right of midline, it is likely that they make some contribution to the localization of acoustic signals containing transient components. Also, binaural sensitivity in the millisecond range may be an important factor in processing latency shifts that arise from IIDs associated with lateralized sound sources (Pollak, 1988; Irvine, 1992).

The injection of kynurenic acid into DNLL clearly alters the ITD sensitivity of neurons in the contralateral inferior colliculus. There is a release from inhibition at every ITD value for which stimulation of the ipsilateral ear produces response suppression. Thus, the dynamic range of ITD curves is compromised, and the sensitivity to binaural time differences is reduced. This result is consistent with our previous investigation of the effects of unilateral kainic acid lesions in DNLL on ITD sensitivity of neurons in the contralateral auditory cortex as reflected by the amplitude of evoked potentials recorded with gross electrodes from the cortical surface (Glenn and Kelly, 1992). The results also support our
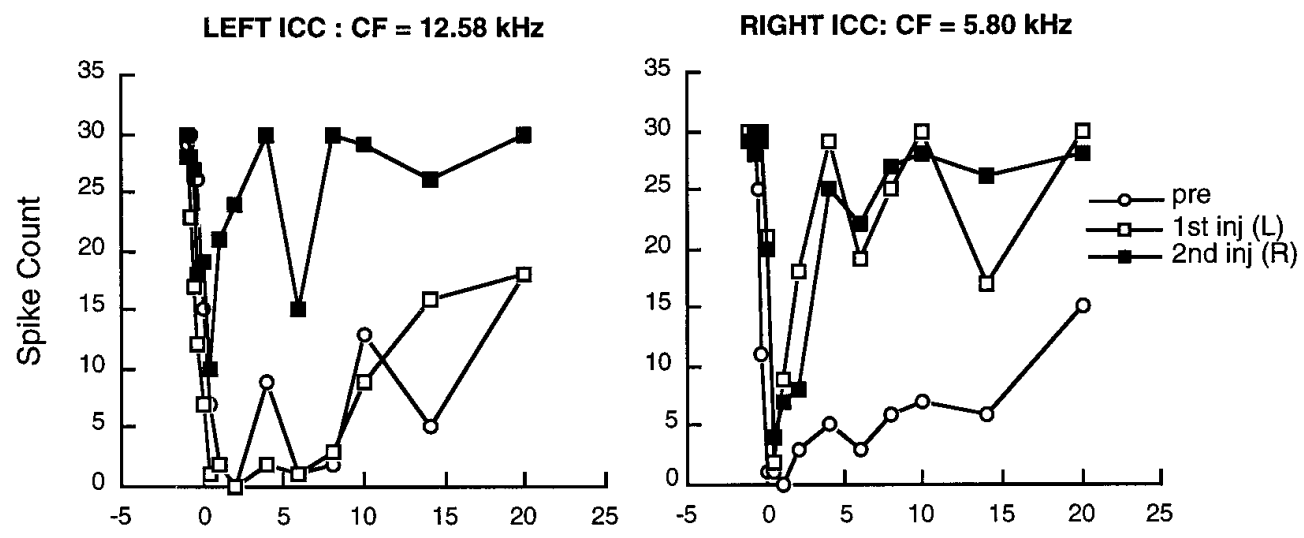

ITD (ipsi re contra, ms.)
Figure 5. The effects of kynurenic acid injections into the left and right DNLL on long-lasting inhibition of responses recorded from two separate neurons in the right and left inferior colliculus of the same rat. The neurons in the left and right inferior colliculus had CFs of 12.58 and $5.80 \mathrm{kHz}$, respectively. Positive values on the $x$-axis represent ipsilateralleading-contralateral ITDs relative to the recording site in inferior colliculus. The responses to ITDs between -1.0 and $+20 \mathrm{msec}$ before LL injection are shown as open circles. The responses after injection of kynurenic acid into the left DNLL are shown as open squares for both neurons. The responses after an injection into the right DNLL are shown as filled squares. 
previous study of the effects of kynurenic acid injections into DNLL on the interaural level sensitivity of single neurons in the inferior colliculus of the rat ( $\mathrm{Li}$ and Kelly, 1992b). Li's study showed that pharmacological blockade of the excitatory synapses in DNLL resulted in an alteration of IID curves obtained from neurons in the contralateral, but not ipsilateral, inferior colliculus. Binaural responses were elicited by paired tone pulses delivered simultaneously to the two ears. After kynurenic acid injection, there was a marked release from the inhibition normally produced by stimulation of the ear ipsilateral to the recording site. Indeed, the Li and Kelly experiment can been viewed as a special case of binaural stimulation with the ITD equal to zero, and the results of both IID and ITD studies can be taken to show that the DNLL contributes to the binaural response of neurons in the contralateral central nucleus of the inferior colliculus.

The disruptive effect on either ITDs or IIDs of injecting kynurenic acid into DNLL suggests that the contralateral projection of this lemniscal nucleus might contribute to sound localization by refining the physiological responses to binaural cues. This expectation has been confirmed by two recent studies of azimuthal sound localization by rats after destruction of the DNLL or its efferent projections. Unilateral or bilateral kainic acid lesions of the DNLL or surgical transection of the commissure of Probst, its exclusive pathway to the contralateral inferior colliculus, DNLL, and other brainstem structures, results in an impairment in sound localization and a degradation in auditory spatial acuity for midline discrimination (Ito et al., 1996; Kelly et al., 1996). Behavioral deficits likely are attributable to abnormalities in processing IIDs, ITDs, or a combination of both.

Intervals $>1.0 \mathrm{msec}$ are clearly beyond the range of ITDs that can serve as cues for sound localization by rats or other mammals, even species with relatively large interaural distances (e.g., the maximum ITD for humans is $\sim 0.6 \mathrm{msec}$ ). Nevertheless, stimulation of the ipsilateral ear in rats produces an inhibition of responses in the inferior colliculus that lasts from 5 to $>20 \mathrm{msec}$, depending on the neuron from which recordings are made (present study). A similar long-lasting inhibition has been reported for neurons in the inferior colliculus of cats and rabbits (Carney and Yin, 1989; Yin, 1994; Fitzpatrick et al., 1995). The present study has shown that the long-lasting inhibition in the inferior colliculus is dependent, at least in part, on synaptic excitation in the contralateral DNLL. Injection of kynurenic acid into DNLL results in a pronounced release from the long-lasting inhibition that is induced by stimulation of the ear ipsilateral to the recording site. The effect is evident over the entire length of the inhibitory period, whether the period lasts 5 or $>20 \mathrm{msec}$. Generalizing from our observations on rats, it seems likely that the DNLL also contributes to the long-lasting inhibition in the inferior colliculus of cats and rabbits, as suggested previously by Carney and Yin (1989), Yin (1994), and Fitzpatrick et al. (1995). Also, the results of brain slice studies by Wu and Kelly (1996) and Fu et al. (1996) suggest that an extended NMDA receptormediated excitatory process in DNLL plays a role in shaping the long-lasting inhibition seen in the inferior colliculus or other structures receiving projections from DNLL. In the brain slice preparation, electrical stimulation of the lateral lemniscus elicits both rapid and sustained excitatory responses that can be blocked by non-NMDA and NMDA antagonists, respectively. The sustained excitation of DNLL neurons would be expected to exert a sustained inhibition on target neurons, provided that the excitatory potentials reached spike threshold.

The functional significance of long-lasting inhibition has not yet been determined by appropriate behavioral studies. However, several investigators have suggested that it might provide a neural substrate for echo suppression, a perceptual phenomenon known as the "precedence effect" in sound localization (Carney and Yin, 1989; Yin, 1994; Fitzpatrick et al., 1995). A similar suggestion has been made regarding long-lasting suppression of neural responses in the DNLL of the mustache bat (Yang and Pollak, 1994 a,b). The time course of the inhibition in inferior colliculus is similar to the time course of the "precedence effect" in both humans and rats (Kelly, 1974). Thus, one possible function of the DNLL might be to suppress neural responses that are associated with multiple sounds (echoes) occurring at delay times between 1 and $20 \mathrm{msec}$.

It should be noted that the echo suppression hypothesis is not incompatible with the suggestion that the DNLL sharpens binaural responses and refines spatial perception of single sound sources by contralateral inhibitory projections that pass through the commissure of Probst (Li and Kelly, 1992b; Ito et al., 1996; Kelly et al., 1996). Blockade of activity in DNLL clearly disrupts both IID and ITD curves in the contralateral inferior colliculus and reduces both short- and long-lasting inhibition produced by stimulation of the ipsilateral ear. The contribution of DNLL to ITD and IID sensitivity is probably important for the localization of single sound sources, whereas the effect of DNLL on longlasting inhibition is more likely to affect the perception of multiple sound sources or reflected sounds (echoes).

In conclusion, the results of our experiment show for the first time that the DNLL plays an active role in shaping the responses of neurons in the central nucleus of the inferior colliculus to interaural time differences. Local injection of kynurenic acid into the contralateral DNLL alters the ITD sensitivity curves of neurons located in the inferior colliculus and greatly reduces the binaural inhibition produced by stimulation of the ipsilateral ear. The release from inhibition is apparent for both short $(0-1 \mathrm{msec})$ and long (1-20 msec) ITD intervals.

\section{REFERENCES}

Adams JC (1979) Ascending projections to the inferior colliculus. J Comp Neurol 183:519-538.

Adams JC, Mugnaini E (1984) Dorsal nucleus of the lateral lemniscus: a nucleus of GABAergic projection neurons. Brain Res Bull 13:585-590.

Bajo VM, Merchán MA, López DE, Rouiller EM (1993) Neuronal morphology and efferent projections of the dorsal nucleus of the lateral lemniscus in the rat. J Comp Neurol 34:241-262.

Beyerl BD (1978) Afferent projections to the central nucleus of the inferior colliculus in the rat. Brain Res 145:209-223.

Brugge JF, Anderson DJ, Aitkin LM (1970) Responses of neurons in the dorsal nucleus of the lateral lemniscus of cat to binaural tonal stimulation. J Neurophysiol 33:441-458.

Brunso-Bechtold JK, Thompson GC, Masterton RB (1981) HRP study of the organization of auditory afferents ascending to the central nucleus of the inferior colliculus. J Comp Neurol 197:705-722.

Buckthought AD (1993) Electrophysiological investigation of the dorsal nucleus of the lateral lemniscus in the auditory system of the albino rat. Master's thesis, Carleton University, Ottawa, Ontario, Canada.

Carney LH, Yin TCT (1989) Responses of low-frequency cells in the inferior colliculus to interaural time differences of clicks: excitatory and inhibitory components. J Neurophysiol 62:144-161.

Coleman JR, Clerici WJ (1987) Sources of projections to subdivisions of the inferior colliculus in the rat. J Comp Neurol 262:215-226.

Covey E (1993) Response properties of single units in the dorsal nucleus of the lateral lemniscus and paralemniscal zone of an echolocating bat. J Neurophysiol 69:842-859.

Faingold CL, Boersma-Anderson CA, Randall ME (1993) Stimulation or blockade of the dorsal nucleus of the lateral lemniscus alters binaural and tonic inhibition in contralateral inferior colliculus neurons. Hear Res 69:98-106. 
Fitzpatrick DC, Kuwada S, Batra R, Trahiotis C (1995) Neural responses to simple simulated echoes in the auditory brain stem of the unanesthetized rabbit. J Neurophysiol 74:2469-2486.

Flammino F, Clopton BM (1975) Neural responses in the inferior colliculus of albino rat to binaural stimuli. J Acoust Soc Am 57:692-695.

Fu XW, Bresden BL, Kelly JB, Wu SH (1996) Synaptic excitation in the dorsal nucleus of the lateral lemniscus: whole-cell patch-clamp recordings from rat brain slice. Neuroscience, in press.

Glenn SL, Kelly JB (1992) Kainic acid lesions of the dorsal nucleus of the lateral lemniscus: effects on binaural evoked responses in rat auditory cortex. J Neurosci 12:3688-3699.

Hutson KA, Glendenning KK, Masterton RB (1991) Acoustic chiasm IV: eight midbrain decussations of the auditory system in the cat. J Comp Neurol 312:105-131.

Irvine DRF (1992) Physiology of the auditory brainstem. In: The mammalian auditory pathway: neurophysiology (Popper AN, Fay RR, eds), pp 153-231. New York: Springer.

Ito M, van Adel B, Kelly JB (1996) Sound localization after transection of the commissure of Probst in the albino rat. J Neurophysiol, in press.

Kelly JB (1974) Localization of paired sound sources in the rat: small time differences. J Acoust Soc Am 55:1277-1284.

Kelly JB, Phillips DP (1991) Coding of interaural time differences of transients in auditory cortex of Rattus norvegicus: implications for the evolution of mammalian sound localization. Hear Res 55:39-44.

Kelly JB, Glenn SL, Beaver CJ (1991) Sound frequency and binaural response properties of single neurons in rat inferior colliculus. Hear Res $56: 273-280$

Kelly JB, Li L, van Adel B (1996) Sound localization after kainic acid lesions of the dorsal nucleus of the lateral lemniscus in the albino rat. Behav Neurosci, in press.

Kudo M (1981) Projections of the nuclei of the lateral lemniscus in the cat: an autoradiographic study. Brain Res 221:57-69.

Li L, Kelly JB (1992a) Binaural responses in rat inferior colliculus following kainic acid lesions of the superior olive: interaural intensity difference functions. Hear Res 61:73-85.

Li L, Kelly JB (1992b) Inhibitory influence of the dorsal nucleus of the lateral lemniscus on binaural responses in the rat's inferior colliculus. J Neurosci 12:4530-4539.

Markovitz NS, Pollak GD (1994) Binaural processing in the dorsal nucleus of the lateral lemniscus. Hear Res 73:121-140.

Merchán MAE, Saldaña E, Plaza I (1994) Dorsal nucleus of the lateral lemniscus in the rat: concentric organization and tonotopic projection to the inferior colliculus. J Comp Neurol 342:259-354.

Moore JK, Moore RY (1987) Glutamic acid decarboxylase-like immunoreactivity in brainstem auditory nuclei of the rat. J Comp Neurol 260:157-174.

Oliver DL, Shneiderman A (1989) An EM study of the dorsal nucleus of the lateral lemniscus: inhibitory, commissural, synaptic connections between ascending auditory pathways. J Neurosci 9:967-982.

Pollak GD (1988) Time is traded for intensity in the bat's auditory system. Hear Res 36:107-124.
Paxinos G, Watson C (1986) The rat brain in stereotaxic coordinates, 2nd Ed. New York: Academic.

Roberts RC, Ribak CE (1987) GABAergic neurons and axon terminals in the brainstem auditory nuclei of the gerbil. J Comp Neurol 258:267-280.

Ross LS, Pollak GD, Zook JM (1988) Origin of ascending projections to an isofrequency region of the mustache bat's inferior colliculus. J Comp Neurol 270:488-505.

Sally SL, Kelly JB (1992) Effects of superior olivary complex lesions on binaural interaction in rat inferior colliculus. Brain Res 572:5-18.

Shneiderman A, Oliver DL (1989) EM autoradiographic study of the projections from the dorsal nucleus of the lateral lemniscus: a possible source of inhibitory inputs to the inferior colliculus. J Comp Neurol 286:28-47.

Shneiderman A, Oliver DL, Henkel C (1988) Connections of the dorsal nucleus of the lateral lemniscus: an inhibitory parallel pathway in the ascending auditory system? J Comp Neurol 276:188-208.

Shneiderman A, Chase MB, Rockwood JM, Benson CG, Potashner SJ (1993) Evidence for a GABAergic projection from the dorsal nucleus of the lateral lemniscus to the inferior colliculus. $J$ Neurochem 60:72-82.

Silverman MS, Clopton BM (1977) Plasticity of binaural interaction. I. Effect of early auditory deprivation. J Neurophysiol 40:1266-1274.

Tanaka K, Otani K, Tokunaga A, Sugita S (1985) The organization of neurons in the nucleus of the lateral lemniscus projecting to the superior and inferior colliculi in the rat. Brain Res 341:252-260.

Thompson GC, Cortez AM, Lam DMK (1985) Localization of GABA immunoreactivity in the auditory brainstem of guinea pigs. Brain Res 339:119-122.

Vater M, Kössl M, Horn AKE (1992) GAD- and GABA-immunoreactivity in the ascending auditory pathway of horseshoe and mustached bats. J Comp Neurol 325:183-206.

Winer JA, Larue DT, Pollak GD (1995) GABA and glycine in the central auditory system of the mustache bat: structural substrates for inhibitory neuronal organization. J Comp Neurol 355:317-353.

Wu SH, Kelly JB (1996) In vitro brain slice studies of the rat's dorsal nucleus of the lateral lemniscus. III. Synaptic pharmacology. J Neurophysiol 75:1271-1282.

Yang L, Pollak GD (1994a) The roles of GABAergic and glycinergic inhibition on binaural processing in the dorsal nucleus of the lateral lemniscus of the mustache bat. J Neurophysiol 71:1999-2013.

Yang L, Pollak GD (1994b) Binaural inhibition in the dorsal nucleus of the lateral lemniscus of the mustache bat affects responses for multiple sounds. Auditory Neurosci 1:1-17.

Yin TCT (1994) Physiological correlates of the precedence effect and summing localization in the inferior colliculus of the cat. $\mathrm{J}$ Neurosci 14:5170-5186.

Zook JM, Casseday JH (1982) Origin of ascending projections to inferior colliculus in the mustache bat, Pteronotus parnellii. J Comp Neurol 207:14-28. 